\title{
SAGES Guidelines for minimally invasive treatment of adrenal pathology
}

\author{
L. Michael Brunt
}

Received: 2 February 2013/Accepted: 2 August 2013/Published online: 31 August 2013

(C) Springer Science+Business Media New York 2013

Minimally invasive adrenalectomy has revolutionized the management of patients with a wide variety of adrenal pathologies. Since its inception, the benefits of this approach have become immediately apparent because open removal of an adrenal tumor, usually small and benign, requires a large painful incision due to the difficult access to the adrenals high in the retroperitoneum.

The Society of American Gastrointestinal and Endoscopic Surgeons (SAGES) Guidelines on Minimally Invasive Approaches to Adrenal Pathology are timely because they takes an evidence-based approach to review where we stand with laparoscopic adrenalectomy in 2013. The Guidelines are comprehensive in their treatment of the various approaches to minimally invasive adrenalectomy and outline important parameters regarding difficult scenarios and perioperative patient management issues.

Adrenal tumors are somewhat unique among surgical conditions for a number of reasons. First, there is a wide variety of tumors that may present with a broad range of clinical presentations, tumor sizes, and imaging characteristics. Second, due to the widespread use of abdominal cross-sectional imaging, the most common adrenal lesion currently is the incidentally discovered adrenal mass, the vast majority of which are benign and do not require surgery. Third, adrenalectomy is an uncommon procedure, and most surgeons in training get little exposure to this procedure, which requires operating in an area that may be less familiar to them anatomically. Finally, the current demographics of the patient population in the United

\section{M. Brunt ( $\square)$}

Section of Minimally Invasive Surgery, Department of Surgery,

Washington University School of Medicine, 660 S Euclid

Avenue, Box 8109, St. Louis, MO 63110, USA

e-mail: bruntm@wustl.edu
States, particularly with regard to the obesity epidemic, add another element to the challenge of these procedures.

It was not the purpose of the SAGES Guidelines to review the clinical presentation and diagnostic evaluation of adrenal tumors or patient selection for adrenalectomy as these topics have been covered extensively in the guideline from the American Association of Endocrine Surgeons and Endocrinologists [1]. However, surgeons who undertake adrenalectomy would behoove themselves to understand the details of diagnostic testing, including biochemical evaluation, and should be familiar with adrenal imaging in order to select patients appropriately for operation and to avoid unnecessary adrenalectomy.

Another unique aspect of adrenalectomy is the variety of operative approaches to the adrenal. The most common are the laparoscopic transabdominal lateral flank approach originally described by Gagner et al. [2] and the retroperitoneal endoscopic approach, which has been perfected and popularized by Walz et al. [3]. Each of these approaches is highly successful in experienced hands, and their advantages and disadvantages have been well covered in the Guidelines.

For the transabdominal approach, which is the most commonly used technique, the key steps to successful adrenalectomy in this author's experience are (1) adequate mobilization of adjacent organs to obtain clear access to the adrenal, which on the right means mobilization of the right lobe of the liver, on the left the splenic flexure of the colon, and together the tail of the pancreas and spleen; (2) use of a gentle, meticulous hemostasis technique and avoidance of aggressive blunt dissection, which can lead to minor bleeding and obscure tissue planes; and (3) careful handling of the adrenal itself and extracapsular dissection to avoid disruption of the tumor capsule and spillage of tumor cells, which can lead to recurrence even with a benign adrenal neoplasm. 
The Guidelines group also discusses newer approaches including robotic and single-incision procedures. As they note, neither of these two techniques has any compelling advantages, and surgeons should await the results of additional studies and higher-level evidence before attempting to adopt them. Certainly, the robotic and singleincision laparoscopic surgery (SILS) approaches can be performed, but should they be performed and at what cost and at what advantage?

The Guidelines also review the literature on a number of challenging scenarios in adrenalectomy including pheochromocytoma, larger tumors, and adrenal cortical carcinoma. Pheochromocytomas can be more difficult to remove because of their larger size and vascularity as well as their potential for location in extraadrenal sites. Large non-pheochromocytoma tumors can likewise be more difficult because of the challenges manipulating the large mass, the potential for effacement or extension behind major vessels such as the vena cava on the right and the renal vein on the left, and the greater potential for malignancy. As a result, they are more likely to result in conversion to open adrenalectomy [4]. To that end, the authors have taken a cautious approach to their recommendations regarding adrenal cortical carcinomas, which in the vast majority of cases because of their large size and recurrence rates should be approached in an open fashion.

One challenging scenario not covered in the Guidelines is that of the obese patient. In this author's experience, obesity is the most common variable that increases the difficulty of both operative exposure in the retroperitoneum and identification of the adrenal within the "sea" of retroperitoneal fat. Patients with Cushing's syndrome may be especially challenging in this regard, and the adrenal may be particularly difficult to locate if the obese patient has a small gland or tumor. In such cases, laparoscopic intraoperative ultrasound may be useful in helping to define the anatomy and location of the adrenal relative to the kidney, pancreas, renal vessels, and vena cava.

Bleeding has been the most common reported complication of adrenalectomy [5]. Recently, however, a number of high-grade complications of adrenalectomy not typically reported in the literature have come to light. These include injuries to the porta hepatis that have resulted in liver failure and have led to liver transplantation, injuries to the renal vessels or ureter requiring nephrectomy, and inappropriate organ resection, particularly mistaken resection of the tail of the pancreas instead of the adrenal gland [6].

Are such complications an inherent risk of an uncommonly performed procedure, or do they reflect inadequate training and experience in performing this operation? The Guidelines group has touched on the issue of the learning curve for adrenalectomy and cites data that suggest a learning curve of $20-40$ cases. Such an experience is almost never obtained during residency training and even rarely within all but a select few fellowship programs. Indeed, for the 2011-2012 academic year, the mean number of adrenalectomy cases managed in residency by graduating chief residents in general surgery according to the Residency Review Committee data for General Surgery was two (which means that many residents likely finished with 0 or 1 adrenal cases). Moreover, no data on numbers for accredited minimally invasive surgery fellowships have been reported.

Previous work from Sosa et al. [7] showed that both surgeon experience and hospital volume may influence the outcome of endocrine surgical procedures. In another study, lower-volume Veterans Association medical centers were found to have higher morbidity and mortality rates after adrenalectomy than university medical centers [8] For an operation that is complex on many levels and entails working in an unfamiliar part of anatomy, these data appear to suggest that most adrenalectomies should be performed in higher-volume centers by surgeons experienced with this operation or interested in it. Certainly, the question of who indeed should be performing adrenalectomy in 2013 needs to be asked. At the least, adrenalectomy in low-volume centers probably should be reserved for lower-risk patients (i.e., those with small tumors, those posing no concern for possible malignancy, and those not morbidly obese).

In summary, the SAGES Guidelines committee is to be commended for their thorough and well-written evidencedbased review of the current literature on this topic. The summary tables at the end of the Guidelines provide a comprehensive look at the outcomes that should provide a benchmark for every surgeon who performs adrenalectomy. In my opinion, these Guidelines will serve as a valuable reference for practicing surgeons as well as residents and fellows in training and should help to frame the most pressing areas for future studies to advance our knowledge and to improve outcomes for this select group of patients.

Disclosure L. Michael Brunt has received honoraria from Ethicon Endo-Surgery, Inc. for teaching and speaking.

\section{References}

1. Zeiger MA, Thompson GB, Duh Q-Y, Hamrahian AH, Angelos P, Elaraj D, Fishman E, Kharlip J (2009) American Association of Clinical Endocrinologists and American Association of Endocrine Surgeons Medical Guidelines for the Management of Adrenal Incidentalomas. Endocr Pract 15(Suppl 1):1-20

2. Gagner M, Lacroix A, Bolte E, Pomp A (1994) Laparoscopic adrenalectomy: the importance of a flank approach in the lateral decubitus position. Surg Endosc 8:135-138 
3. Walz M, Wenger F, Piero A et al (2006) Posterior retroperitoneoscopic adrenalectomy: results of 560 procedures in 520 patients. Surgery 140:943-948

4. Bittner JB, Gershuni V, Matthews BD, Moley JF, Brunt LM (2013) Risk factors affecting operative approach, conversion, and morbidity for adrenalectomy: a single-institution series of 402 patients. Surg Endosc 27(7):2342-2350

5. Brunt LM (2006) Minimal access adrenal surgery. Surg Endosc 20:351-361

6. Tessier DJ, Iglesias R, Chapman WC, Kercher KW, Matthews BD, Gorden L, Brunt LM (2009) Previously unreported high-grade complications of adrenalectomy. Surg Endosc 23:97-102
7. Sosa JA, Bowman HM, Tielsch JM, Powe NR, Gordon TA, Udelsman R (1998) The importance of surgeon experience for clinical and economic outcomes from thyroidectomy. Ann Surg 228:320-330

8. Turrentine FE, Henderson WG, Khuri SF, Schiffnet TL, Inabnet WB (2007) Adrenalectomy in Veterans Affairs and selected university medical centers: results of the patient safety in surgery study. J Am Coll Surg 204:1273-1293 